\title{
VIRTUAL HORSEBACK ARCHERY
}

\author{
Kibakiba Mushamusha
}

Masataka Imura, Jun Kozuka, Koichi Minami, Yoshito Tabata, Tatsuya

Shuzui and Kunihiro Chihara

Graduate School of Information Science, Nara Institute of Science and Technology

imura@is.aist-nara.ac.jp

\begin{abstract}
A horseback archery (called Yabusame in Japanese) is the traditional art of Japan. In this project the authors recreate a horseback archery in simple style utilizing fusion of various sensors and movable L-shape immersive screens. The developed system enables ordinary people to enjoy the excitement of a horseback archery.
\end{abstract}

Keywords: Horseback archery, virtual reality, and immersive display

\section{Introduction}

A horseback archery (called Yabusame in Japanese) is the traditional art that dates from the Heian Era (10th century). Today, a horseback archery takes place in shrines as an event of yearly fortune telling. A player who is dressed in costume of samurai urges a horse on and hits targets one after another by shooting arrows (Figure 1[1]).

Since a horseback archery requires both horse riding skill and archery skill simultaneously, ordinary people cannot experience this excitement during their lifetimes. The authors extract essential elements from a horseback archery and reconstruct them in more simple style utilizing virtual reality technologies.

Over the past twenty years, a considerable number of sensory video game machines have been released one after another. However, only few game machines simulate horse riding. "Final furlong" (Namco)[2] is one of these rare games and provide users with the feeling as if they were leading jockeys. Nevertheless, there has been no attraction combining horse riding and shooting, to say nothing of a horseback archery. On the other hand, some foregoing researches adopted virtual horseback riding

The original version of this chapter was revised: The copyright line was incorrect. This has been corrected. The Erratum to this chapter is available at DOI: 10.1007/978-0-387-35660-0_65 


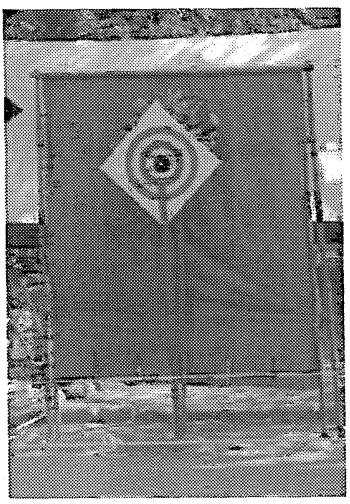

(a)

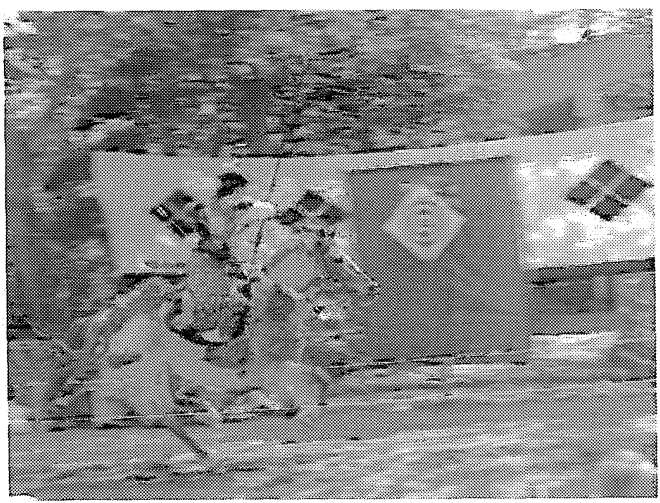

(b)

Figure 1. Japanese horseback archery (a) target (b) shooting

for rehabilitation exercises[3][4]. However, these researches didn't bring entertainment into view.

In this project, the authors recreate a horseback archery utilizing fusion of various sensors and movable L-shape immersive screens. To improve quality as entertainment, the authors import competition between two players into the traditional style of horseback archery.

\section{System}

The authors consider that the excitement of a horseback archery comes from two points: speed of horse riding and hits to moving targets. The attraction Kibakiba Mushamusha has been developed placing emphasis on these two points and other trivial factors are sometimes neglected by design.

\subsection{Booth Setting}

A player sits on a simple rocking chair that imitates a horseback. The rocking chair is anchored to a floor and is allowed to swing only back and forth. Two screens $(2 \mathrm{~m} \times 2 \mathrm{~m})$ are set in L-shape, one is in front of the player and the other is on the left side.

\subsection{Flow of Game}

A player's back-and-forth swing on a horseback makes a horse go ahead. As the horse runs, targets come in sight one after another. A player shoots the targets with a bow and arrow. When a player shoots 


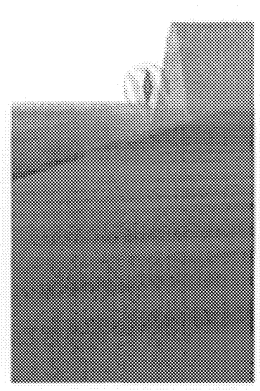

(a)

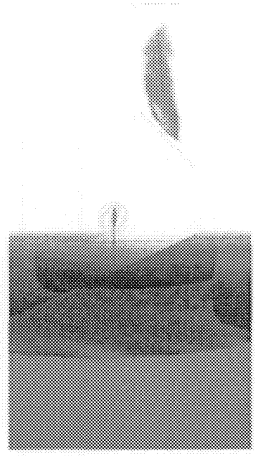

(b)

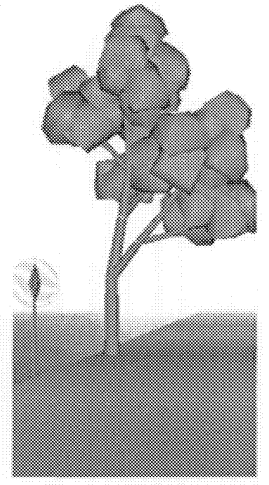

(c)

Figure 2. Obstacles born by hitting targets (a) water (b) fire (c) tree

a real arrow, a virtual arrow flies with the initial condition that reflects the position and orientation of the real arrow.

Two players on a horseback run side by side and targets are located at almost even intervals between them. Therefore, to get on the game advantageously, a player must urge a horse faster than an opponent and shoot as many targets as possible. The player falling behind must shoot the small targets that the precedent player missed to shoot. However, faster running makes it difficult to aim targets accurately. Consequently, a player must keep balance between horse riding and arrow shooting.

Targets differ from each other in size, height and attribute. Each target has one of three attributes: water, fire and tree. The attribute represents a type of obstacle which appears in front of the opponent when a player hits the target (Figure 2). A player can shoot the opponent also. If a player is hit by the arrow, the horse rises on its hind legs and stops.

A player who got higher points in a fixed period becomes a winner. the higher point is assigned to the smaller target. Usual game period is 100 seconds.

\section{Imprementation}

The block diagram of the system is shown in Figure 3. The system consists of five computers. One gathers data concerning players' motion from sensors and others render scenes consistently. Figure 4 shows the arrangement of players and apparatus. To pack all apparatus compactly (in $6 \mathrm{~m} \times 6 \mathrm{~m}$ square), projectors are located in the way that light paths of two projectors cross each other. 


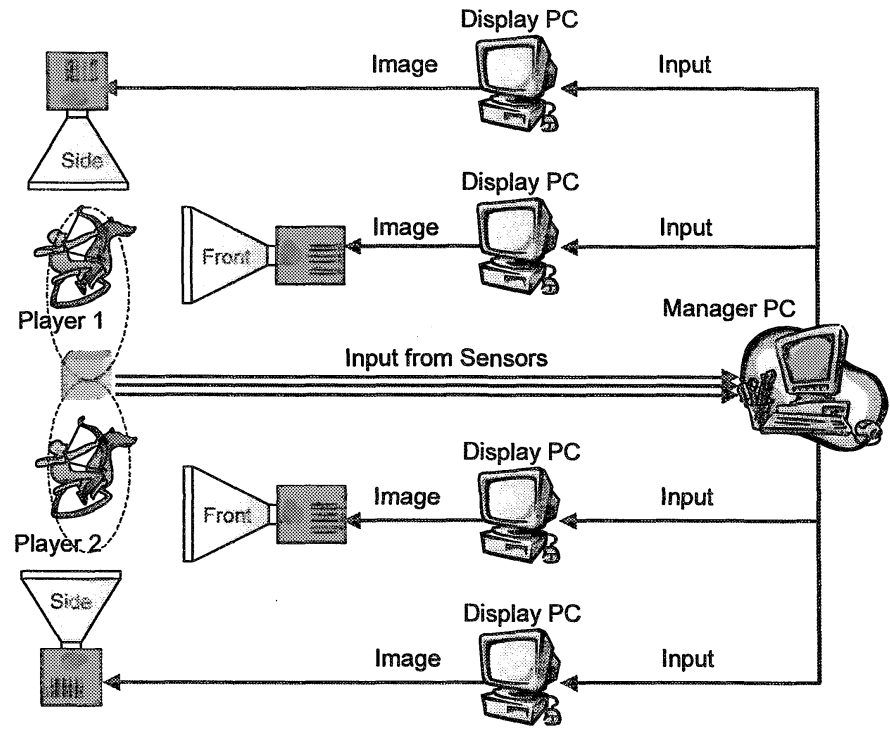

Figure 3. Block diagram of system

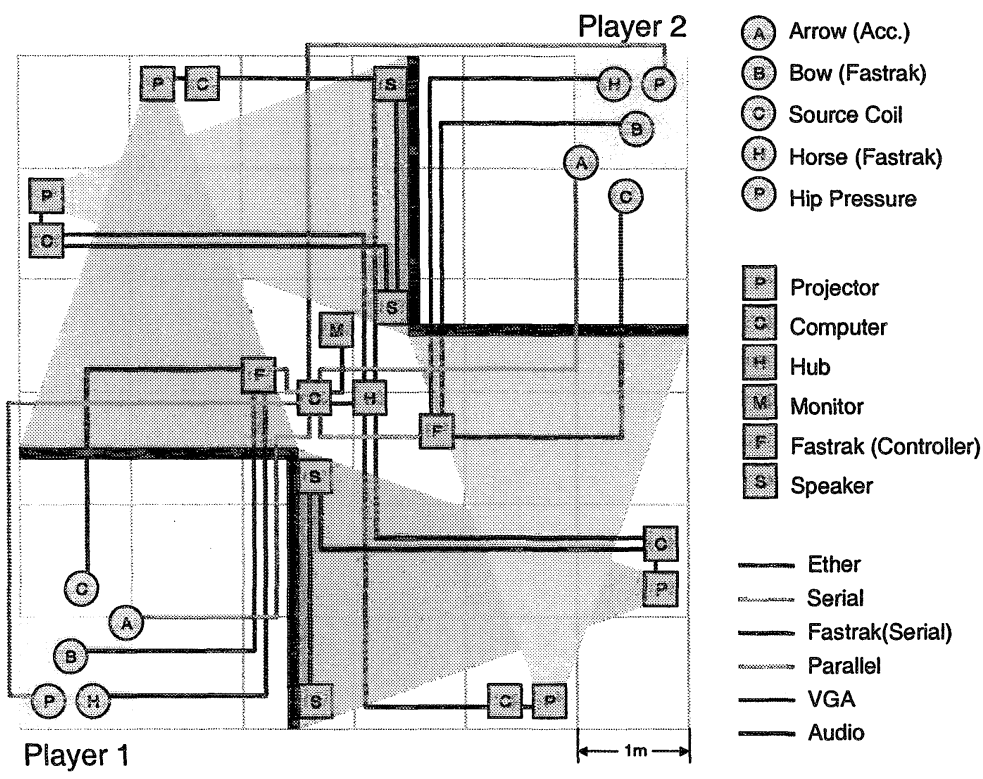

Figure 4. Arrangement of players and apparatus 


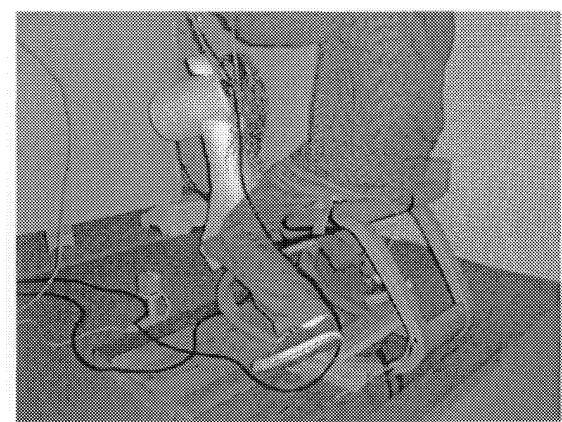

Figure 5. Horse
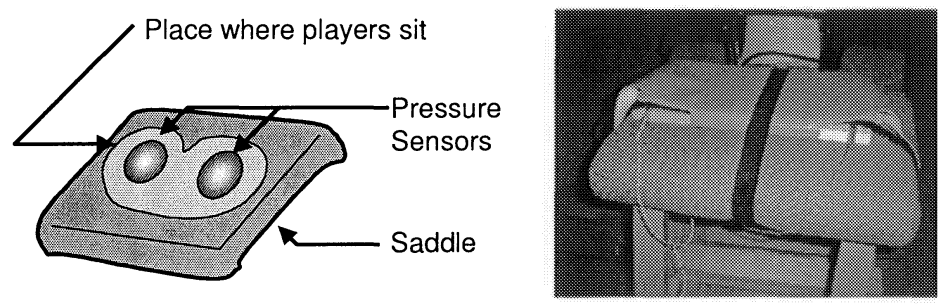

Figure 6. Sensing of center of balance

\subsection{Horse}

A horse consists of a wooden rocking chair and a head that makes of expanded polystyrene (Figure 5). The chair and the head are hinged and supported by spring and rubber sheet. A rocking chair is suitable for reproducing the swing on a horseback. On the tip of the nose, a positional sensor (Polhemus Fastrak) is attached and measures the movement of the horse. Frequency component of the movement is calculated by fast Fourier transformation of time series of position and the velocity of the horse is decided using leak integrate fire model[5] from neural network theory. Each neuron has frequency component of time series of position as input and accelerates the horse when sum of input exceeds a certain level. This process simulates the behavior of horse which is sometimes unlikely to run or to stop as we wish.

On the saddle, two pressure sensors are located under the cover (Figure 6). These sensors are used to measure the movement of a center of balance. If the left pressure is higher than right one, it is estimated that a player leans to the left. This information is used to control the course of a horse. 


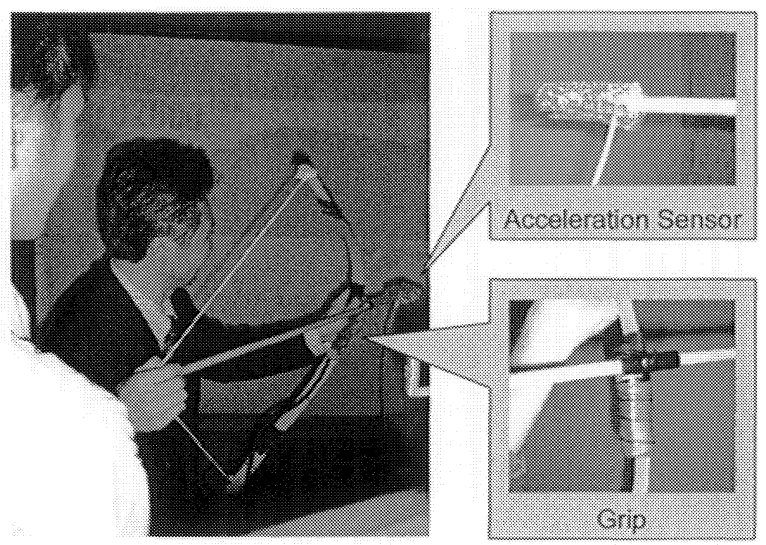

Figure 7. Bow and arrow

\subsection{Bow and Arrow}

The positional sensor same as the one on the nose of the horse is also attached on the bow and measures the position and direction of the bow. To make it easy for players to shoot an arrow, the arrow is put through a pipe which is tied up the bow. On the tip of the arrow, an acceleration sensor is attached (Figure 7). From rapid change of acceleration, the release of an arrow is detected. To avoid consecutive shoots by one release, another shoot is prohibited for one second after shooting one arrow.

\subsection{Movable Screen}

The L-shape screen is made from aluminum pipes and shoji (Japanese sliding-screen) paper. The scene is projected from back side of the screen by portable liquid crystal projectors. The screen can be disassembled into parts and carried handily, therefore we can construct the screen anywhere and generate the virtual environment with ease.

The size of shoji paper is $2 \mathrm{~m} \times 4 \mathrm{~m}$ and folded in the center. To gain the higher immersion to virtual environment, the seam of two screens (in this case, front and left) must be inconspicuous. For this purpose, the centerfold is pinned to the pipes with clothes pegs from the backside of screen.

\subsection{Procedure}

The procedure in one frame of the game is as follows. 
1 A server computer gathers the data of players' movement from various sensors (magnetic positional sensors, acceleration sensors and pressure sensors).

2 The server delivers the input data to four client computers.

3 Each client computer calculates the movement of objects. As even random number generation is controlled, four results of calculation are completely same. After that, each computer renders the scene from its own viewpoint.

4 When clients finish rendering the scene, they send a signal to the server.

5 When server receives the signals from all clients, the server gives clients the signal to flip display buffer so that all screens are updated simultaneously.

This structure enables us to reconfigure the number of client computers, i.e. the number of screens. Slight change of configuration parameters enables this application to be ready for various screen arrangements.

\subsection{Realization of Even Condition}

In Japan, it is said that nine out of ten people are right-handed. For right-handed people, to shoot an arrow to the left is easier than to the right, and the reverse is also true. In the virtual world, targets are located between two players, so one player becomes advantageous to another. Therefore, the whole world which the second player sees is inverted like a reflection in a mirror so as to make the conditions of both players even.

\section{Result}

In December, 2000, this attraction took part in the 8th Intercollegiate Virtual Reality Contest[6] which was held in Gifu, Japan and got the second prize (Gifu VR grand prize) out of five participants. Figure 8 shows playing scenes in the contest.

In August, 2001, this attraction was exhibited in Fukusaki Exciting Virtual Square for Children (at Osaka, Japan). The aim of this event is to let handicapped children who cannot even go outdoors experience various exciting worlds through virtual reality applications. For one week, lots of children enjoyed playing this system. 

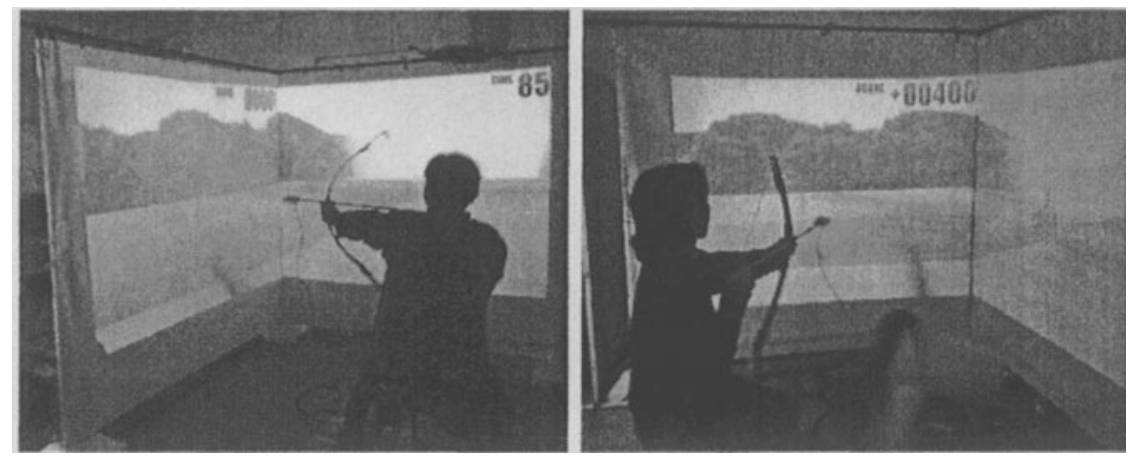

Figure 8. Result

\section{Conclusion}

The authors developed a virtual horseback archery, named Kibakiba Mushamusha, utilizing fusion of various sensors and movable L-shape immersive screens. The system reproduced Japanese traditional horseback archery in more familiar style and enabled ordinary people to enjoy the excitement of a horseback archery easily.

\section{Acknowledgements}

A part of this attraction was developed under the support of the committee of Intercollegiate Virtual Reality Contest and the committee of Fukusaki Exciting Virtual Square for Children.

\section{References}

[1] O. Terasaki, "Iza! KAMAKURA", http://kamakura.cool.ne.jp/tera_k1/kamakura/

[2] Namco Ltd., "Final Furlong", http://www.namco.co.jp/home/aa/am/vg/finalfurlong/

[3] R. Kijima et al., "A Development of VR KARAKURI Horse Riding System for Exhaustive Therapy and Study on its Validity", Transactions of the Virtual Reality Society of Japan, Vol. 6, No. 3, pp. 157-164, 2001.

[4] Y. Shinomiya et al., "Horseback Riding Therapy Simulator with VR Technology", ACM Symposium on Virtual Reality Software and Technology, pp. 9-14, 1997.

[5] T. Shimokawa, et al., "A First-passage-time Analysis of the Periodically Forced Noisy Leaky Integrate-and-fire Model", Biological Cybernetics, Vol. 83, pp. 327$340,2000$.

[6] The 8th Intercollegiate Virtual Reality Contest, http://www.ihl.t.u-tokyo.ac.jp/IVRC/2000/ 